\title{
Sharp Gagliardo-Nirenberg Inequalities via $p$-Laplacian Type Equations
}

\author{
Martial Agueh
}

\begin{abstract}
Sharp constants and optimal functions of Gagliardo-Nirenberg inequalities involving the $L^{p}$-norm of the gradient are investigated by studying a $p$-Laplacian type equation. By transforming the unknown of the equation via some change of function, we find explicitly the solution of this equation in all generalities when the space dimension is $n=1$, and in some particular cases when $n>1$. Then we derive the sharp constants and optimal functions of all the $L^{p}$ Gagliardo-Nirenberg inequalities when $n=1$, and of a subclass of them when $n>1$. Our analysis includes also the sharp $L^{p}$ Nash's inequalities.
\end{abstract}

Mathematics Subject Classification (2000). 39B62, 35J20, 34A34, 34A30.

Keywords. Gagliardo-Nirenberg inequalities, Nash's inequalities, best constants, optimal functions, $p$-Laplacian type equations.

\section{Introduction}

The present work extends ideas presented in our previous papers [1] and [2] to Gagliardo-Nirenberg inequalities [9,12] involving the gradient $L^{p}$-norm. In [1] the author explored the link between mass transport theory and Gagliardo-Nirenberg inequalities. He showed that in dimension $n=1$, the best constants and optimal functions of all the $L^{2}$ Gagliardo-Nirenberg inequalities can be computed via a linear first order ordinary differential equation (ODE), which is obtained from the Euler-Lagrange equation of the variational problem leading to these inequalities through some specific change of function. And using the solutions to this ODE, the author was able to re-establish the link between mass transport theory and certain Gagliardo-Nirenberg inequalities, first proved in [6]. In [2], the author extended the above ideas to the $L^{2}$ Gagliardo-Nirenberg inequalities in higher dimensional spaces, $n>1$. There, he showed that if $n>1$, then the ODE giving

The author is supported by a grant from the Natural Science and Engineering Research Council of Canada. 
the best constants and optimal functions of the Gagliardo-Nirenberg inequalities is rather nonlinear, but for a one-parameter family of the $L^{2}$ Gagliardo-Nirenberg inequalities (namely $q=1+\frac{s}{2}$ or $q=2(s-1$ ) in inequality (1) below), it reduces to a linear ODE which he solved explicitly. In these cases, he recovers results previously obtained by Del-Pino and Dolbeault in [7]. In this paper, we generalize the analysis of [1] and [2] to the $L^{p}$ Gagliardo-Nirenberg inequalities and $L^{p}$ Nash's inequalities, for all $p>1$ and all space dimensions $n \geq 1$, that is,

$$
\|u\|_{L^{s}\left(\mathbb{R}^{n}\right)} \leq K_{\text {opt }}\|\nabla u\|_{L^{p}\left(\mathbb{R}^{n}\right)}^{\theta}\|u\|_{L^{q}\left(\mathbb{R}^{n}\right)}^{1-\theta} \quad \forall u \in D^{p, q}\left(\mathbb{R}^{n}\right),
$$

where $\theta=\frac{n p(s-q)}{s[n p-q(n-p)]}, K_{o p t}>0$,

$$
D^{p, q}\left(\mathbb{R}^{n}\right):=\left\{u \in L^{q}\left(\mathbb{R}^{n}\right): \nabla u \in L^{p}\left(\mathbb{R}^{n}\right)\right\}
$$

and

$$
n>1, \quad 1<p<n, \quad \text { and } \quad 1 \leq q<s<\bar{p}:=\frac{n p}{n-p}
$$

or

$$
n=1, \quad p>1 \text { and } 1 \leq q<s<\infty .
$$

As mentioned in [8], (see also Section 2 of this manuscript), one way to obtain the best constant $K_{\text {opt }}$ and optimal functions of the Gagliardo-Nirenberg/Nash's inequality (1) is to determine an explicit minimizer $u_{\infty}$ of the variational problem

$$
\inf \left\{E(u):=\frac{1}{p} \int_{\mathbb{R}^{n}}|\nabla u|^{p} \mathrm{~d} x+\frac{1}{q} \int_{\mathbb{R}^{n}}|u|^{q} \mathrm{~d} x: \quad u \in D^{p, q}\left(\mathbb{R}^{n}\right), \quad\|u\|_{L^{s}\left(\mathbb{R}^{n}\right)}=1\right\} .
$$

This requires solving explicitly the $p$-Laplacian type equation

$$
-\operatorname{div}\left(|\nabla u|^{p-2} \nabla u\right)+|u|^{q-2} u=\lambda|u|^{s-2} u,
$$

that is, the Euler-Lagrange equation to problem (2), where $\lambda$ denotes the Lagrange multipliers for the constraint $\|u\|_{L^{s}\left(\mathbb{R}^{n}\right)}=1$. By a routine rearrangement argument (see $[4,14]), u_{\infty}(x)$ can be chosen non-negative, radially-symmetric, nonincreasing, and tends to 0 as $|x|$ tends to $\infty$. Invoking Serrin-Tang [13], we see that $u_{\infty}$ is the unique radial, non-negative and non-trivial smooth solution of PDE (3) that tends to 0 at $\infty$. Then, there exists a nonincreasing function $v:[0, \infty) \rightarrow[0, \infty)$ with $\lim _{r \rightarrow \infty} v(r)=0$, such that

$$
u_{\infty}(x)=v(r), \quad r=|x| .
$$

This implies that

$$
\frac{r^{p^{*}}}{p^{*}}=H(v(r))=H\left(u_{\infty}(x)\right),
$$

where $H=(v \circ g)^{-1}, g(t)=\left(p^{*} t\right)^{1 / p^{*}}$ and $\frac{1}{p}+\frac{1}{p^{*}}=1$. The above argument suggests that we introduce the change of function

$$
H(u(x))=\frac{r^{p^{*}}}{p^{*}}, \quad r=|x|,
$$


to solve PDE (3). Another argument that justifies the change of function (5) relies on the link between mass transport theory and certain Gagliardo-Nirenberg inequalities, as explained in [1]. Indeed, from mass transport theory, one can establish the following duality (see $[3,6]$ ):

$$
\begin{aligned}
\sup \left\{-H_{|x|^{p^{*}} / p^{*}}^{F}\left(\rho_{1}\right), \rho_{1} \in \mathcal{P}\left(\mathbb{R}^{n}\right)\right\}= & \inf \left\{J_{F}\left(\rho_{0}\right):=-H^{F+n P_{F}}\left(\rho_{0}\right)\right. \\
& \left.+\frac{1}{p} \int_{\mathbb{R}^{n}}\left|\nabla\left(F^{\prime}\left(\rho_{0}\right)\right)\right|^{p} \rho_{0} \mathrm{~d} x, \rho_{0} \in \mathcal{P}\left(\mathbb{R}^{n}\right)\right\},
\end{aligned}
$$

and optimality in both problems is achieved at the probability density $\rho_{0}=\rho_{1}:=$ $\rho_{\infty}$, solution of the ODE

$$
\nabla\left(F^{\prime}\left(\rho_{\infty}\right)+\frac{|x|^{p^{*}}}{p^{*}}\right)=0
$$

Here $F:[0, \infty) \rightarrow \mathbb{R}$ satisfies $F(0)=0$ and $x \mapsto x^{n} F\left(x^{-n}\right)$ is convex and nonincreasing (see McCann [11]), $P_{F}(x)=x F^{\prime}(x)-F(x), H_{|x|^{p^{*}} / p^{*}}^{F}(\rho)=H^{F}(\rho)+$ $H_{|x|^{p^{*}} / p^{*}}(\rho)=\int_{\mathbb{R}^{n}} F(\rho(x)) \mathrm{d} x+\int_{\mathbb{R}^{n}} \frac{|x|^{p^{*}}}{p^{*}} \rho(x) \mathrm{d} x$, and $\mathcal{P}\left(\mathbb{R}^{n}\right)$ denotes the set of probability densities on $\mathbb{R}^{n}$ with finite $p^{*}$-moment. To obtain the GagliardoNirenberg inequality (1) from the duality (6), it is sufficient to choose conveniently $\rho_{0}=\psi(u)$ and the function $F$, so that $J_{F}\left(\rho_{0}\right)=J_{F}(\psi(u))$ coincides - up to some multiplicative constants - to the functional $E(u)$ in (2); for example, if $q=1+\frac{s}{p^{*}}$, then one can choose $\psi(u)=u^{s}$ and $F(x)=-x^{\frac{1}{s}+\frac{1}{p^{*}}}$. Once this choice is made, then the solution $\rho_{\infty}$ to $\operatorname{ODE}(7)$ determines the solution $u_{\infty}$ to PDE (3), via the transformation $u_{\infty}=\psi^{-1}\left(\rho_{\infty}\right)$. In other words, $u_{\infty}$ is the solution of

$$
F^{\prime}\left(\psi\left(u_{\infty}\right)\right)+\frac{|x|^{p^{*}}}{p^{*}}=\gamma,
$$

or equivalently

$$
\frac{r^{p^{*}}}{p^{*}}=\frac{|x|^{p^{*}}}{p^{*}}=\gamma-F^{\prime}\left(\psi\left(u_{\infty}\right)\right):=H\left(u_{\infty}(x)\right),
$$

where $\gamma$ is a constant. So it appears that to determine an explicit minimizer of the functional $J_{F}\left(\rho_{0}\right)=J_{F}(\psi(u))=E(u)$, one need to find a function $H$ that satisfies (9), that is also (5). And note that after finding $H$, one can use again (9) to look for the appropriate functions $\psi$ and $F$ required in order to link mass transport theory to the Gagliardo-Nirenberg/Nash's inequality (1) via the duality (6). In this work, we show with the help of the change of function (5), that PDE (3) leads to the nonlinear first order ODE in $G^{\prime}(t)=\frac{\left|H^{\prime}(t)\right|^{p-2} H^{\prime}(t)}{p-1}$,

$$
p\left(\frac{t^{q}}{q}-\frac{t^{s}}{s}\right) G^{\prime \prime}(t)+(p-1)\left(t^{q-1}-t^{s-1}\right) G^{\prime}(t)-\frac{p(n-1)}{p-1} G^{\prime \prime}(t) \int_{0}^{t} \frac{\mathrm{d} \tau}{G^{\prime}(\tau)}=n,
$$

whose solution $G^{\prime}$ determines explicitly a minimizer $u_{\infty}$ to problem (2) (see Theorem 3.1). If $n=1$, then ODE (10) reduces to a linear first order ODE in $G^{\prime}$. In 
this case, we obtain the best constants and optimal functions of the $L^{p}$ GagliardoNirenberg/Nash's inequalities on the real line, for all values of $p, q$ and $s$. If $n>1$, due to the nonlinear term $\frac{p(n-1)}{p-1} G^{\prime \prime}(t) \int_{0}^{t} \frac{\mathrm{d} \tau}{G^{\prime}(\tau)}$, we have not been able to solve ODE (10) explicitly, in all generalities. But we observe that if we furthermore assume that $G^{\prime \prime}(t) \int_{0}^{t} \frac{\mathrm{d} \tau}{G^{\prime}(\tau)}$ is a constant, then (10) reduces to a linear ODE which can be solved explicitly. In this case, we recover the sharp constants and optimal functions of the $L^{p}$ Gagliardo-Nirenberg inequalities previously obtained in [8] when $q=1+\frac{s}{p^{*}}$ or $q=p^{*}(s-1)$ and $n \geq 2$. To our knowledge, this is the first work where best constants and optimal functions of all the $L^{p}$ GagliardoNirenberg inequalities are obtained on the real line. One of the advantages of our method, compared with the direct mass transport method, is that it does not require choosing a priori a function $F$ in the duality (6). Actually, this choice is not known when $q \neq 1+\frac{s}{p^{*}}$ and $q \neq p^{*}(s-1)$, not even on the real line. Instead, we expect that our method will help in finding the right function $F$ needed in the duality (6) for the remaining $L^{p}$ Gagliardo-Nirenberg inequalities to be recovered from mass transport theory. This could also lead to new examples of displacement convex functionals $H^{F}$, which are proved very useful in applications of mass transport theory to other fields of research.

The paper is organized as follows. In Section 2, we derive the best constant and optimal functions of the Gagliardo-Nirenberg/Nash's inequality (1) in terms of a minimizer $u_{\infty}$ of the variational problem (2). In Section 3, we establish the ODE (10) that determines a minimizer $u_{\infty}$ to (2), and we solve this ODE for all values of $p, q$ and $s$ when $n=1$, and for some particular cases when $n>1$. Then we obtain the best constants and optimal functions of all the $L^{p}$ GagliardoNirenberg inequalities when $n=1$, and of a subclass of them when $n>1$. Finally, we point out that our analysis also allows to obtain the best constants and optimal functions of the $L^{p}$ Nash's inequalities, at least on the real line, though these results are already known in the literature (see [5]).

Throughout the paper, $\|u\|_{q}$ denotes the $L^{q}$-norm of a function $u: \mathbb{R}^{n} \rightarrow \mathbb{R}$, $\operatorname{sign}(u)$ stands for the sign of $u, \chi_{A}$ denotes the characteristic function a set $A$, and $\bar{p}:=n p /(n-p), p^{*}:=p /(p-1)$ are respectively the Sobolev and conjugate exponents of $p$.

\section{Best constants in Gagliardo-Nirenberg/Nash's inequalities}

In this section, we find the best constants and optimal functions of the $L^{p}$ Gagliardo-Nirenberg and $L^{p}$ Nash's inequalities, assuming that the variational problem

$$
\inf \left\{E(u):=\frac{1}{p} \int_{\mathbb{R}^{n}}|\nabla u|^{p} \mathrm{~d} x+\frac{1}{q} \int_{\mathbb{R}^{n}}|u|^{q} \mathrm{~d} x: u \in D^{p, q}\left(\mathbb{R}^{n}\right),\|u\|_{L^{s}\left(\mathbb{R}^{n}\right)}=1\right\}
$$

has a minimizer $u_{\infty}$. The proof of existence of a minimizer to this problem is standard (see for example [8]). 
Theorem 2.1. Let $n, p, q$ and $s$ be such that

$$
\begin{aligned}
& 1<p<n \quad \text { and } \quad 1 \leq q<s<\bar{p}:=\frac{n p}{n-p} \quad \text { if } \quad n>1 \\
& p>1 \quad \text { and } \quad 1 \leq q<s<\infty \quad \text { if } n=1 \text {. }
\end{aligned}
$$

Assume that the variational problem

$$
\inf \left\{E(u):=\frac{1}{p} \int_{\mathbb{R}^{n}}|\nabla u|^{p} d x+\frac{1}{q} \int_{\mathbb{R}^{n}}|u|^{q} d x: u \in D^{p, q}\left(\mathbb{R}^{n}\right),\|u\|_{L^{s}\left(\mathbb{R}^{n}\right)}=1\right\}
$$

has a minimizer $u_{\infty}$. Then the $L^{p}$ Gagliardo-Nirenberg/Nash's inequality

$$
\|u\|_{L^{s}\left(\mathbb{R}^{n}\right)} \leq K_{\text {opt }}\|\nabla u\|_{L^{p}\left(\mathbb{R}^{n}\right)}^{\theta}\|u\|_{L^{q}\left(\mathbb{R}^{n}\right)}^{1-\theta} \quad \forall u \in D^{p, q}\left(\mathbb{R}^{n}\right)
$$

holds with $\theta=\frac{n p(s-q)}{s[n p-q(n-p)]}$, and the sharp constant $K_{\text {opt }}>0$ is explicitly given by

$$
K_{\text {opt }}=\left[\frac{K(n, p, q, s)}{E\left(u_{\infty}\right)}\right]^{\frac{n p+p s-n q}{s[n p-q(n-p)]}}
$$

where

$$
K(n, p, q, s)=\frac{\alpha+\beta}{(q \alpha)^{\frac{\alpha}{\alpha+\beta}}(p \beta)^{\frac{\beta}{\alpha+\beta}}}, \quad \alpha=n p-s(n-p), \quad \beta=n(s-q) .
$$

Moreover, $u_{\sigma, \bar{x}}(x)=C u_{\infty}(\sigma(x-\bar{x}))$ are optimal functions in inequality (13), for arbitrary $C \neq 0, \sigma \neq 0$ and $\bar{x} \in \mathbb{R}^{n}$.

Proof. It follows the lines of the proof of Theorem 2.1 in [2]. Indeed, since $u_{\infty}$ is a minimizer of (12), we have for all $0 \neq u \in D^{p, q}\left(\mathbb{R}^{n}\right)$

$$
E\left(u_{\infty}\right) \leq E\left(\frac{u}{\|u\|_{s}}\right)=\frac{\|\nabla u\|_{p}^{p}}{p\|u\|_{s}^{p}}+\frac{\|u\|_{q}^{q}}{q\|u\|_{s}^{q}},
$$

with equality if $u=u_{\infty}$. Using the scaled function $u_{\lambda}(x)=u\left(\frac{x}{\lambda}\right)$ in (16), we have that

that is,

$$
E\left(u_{\infty}\right) \leq \frac{\lambda^{n-p-\frac{n p}{s}}}{p} \frac{\|\nabla u\|_{p}^{p}}{\|u\|_{s}^{p}}+\frac{\lambda^{n\left(1-\frac{q}{s}\right)}}{q} \frac{\|u\|_{q}^{q}}{\|u\|_{s}^{q}}
$$

$$
E\left(u_{\infty}\right) \leq \lambda^{n-p-\frac{n p}{s}} A+\lambda^{n\left(1-\frac{q}{s}\right)} B:=f(\lambda),
$$

for all $0 \neq u \in D^{p, q}\left(\mathbb{R}^{n}\right)$ and $\lambda>0$, where $A=\frac{\|\nabla u\|_{p}^{p}}{p\|u\|_{s}^{p}}$ and $B=\frac{\|u\|_{q}^{q}}{q\|u\|_{s}^{q}}$. From the assumption (11), we see that $n-p-\frac{n p}{s}<0$ and $n\left(1-\frac{q}{s}\right)>0$. Then the minimum over $\lambda>0$ of $f(\lambda)$ is attained at

$$
\lambda_{\min }=\left[\frac{\left(p-n+\frac{n p}{s}\right) A}{n\left(1-\frac{q}{s}\right) B}\right]^{\frac{s}{n p+p s-n q}} .
$$

Therefore, by a direct computation, (17) gives

$$
E\left(u_{\infty}\right) \leq f\left(\lambda_{\min }\right)=K(n, p, q, s) \frac{\|\nabla u\|_{p}^{\frac{n p(s-q)}{n p+p s-n q}}\|u\|_{q}^{\frac{q[n p-s(n-p)]}{n p+p s-n q}}}{\|u\|_{s}^{\frac{s[n p-q(n-p)]}{n p+p s-n q}}}
$$


that is,

$$
\|u\|_{s} \leq\left[\frac{K(n, p, q, s)}{E\left(u_{\infty}\right)}\right]^{\frac{n p+p s-n q}{s[n p-q(n-p)]}}\|\nabla u\|_{p^{\frac{n p(s-q)}{s[n p-q(n-p)]}}\|u\|_{q}^{\frac{q[n p-s(n-p)]}{s[n-q(n-p)]}}}
$$

where

$$
K(n, p, q, s)=\frac{1+\gamma_{0}}{\left(q \gamma_{0}\right)^{\frac{n p-s(n-p)}{n p+p-n q}} p^{\frac{n(s-q)}{n p+p s-n q}}}, \quad \gamma_{0}:=\frac{n p-s(n-p)}{n(s-q)} .
$$

Inequality (20) reads as the Gagliardo-Nirenberg/Nash's inequality (13), where $\theta=\frac{n p(s-q)}{s[n p-q(n-p)]}$, and the sharp constant $K_{o p t}$ is given by (14). Now, we set $\alpha=n p-s(n-p)$ and $\beta=n(s-q)$ in $(21)$. We have that $\gamma_{0}=\frac{\alpha}{\beta}$ and $n p+p s-$ $n q=\alpha+\beta$, and then (21) reads as (15). Finally, from our analysis, it appears that any minimizer $u_{\infty}$ of the variational problem (12) is an optimal function in inequality (13). And since (13) is invariant under translation $x \mapsto x-\bar{x}$, scaling $x \mapsto \sigma x$, and multiplication by a constant $u \mapsto C u$, we deduce that $u_{\sigma, \bar{x}}(x)=$ $C u_{\infty}(\sigma(x-\bar{x}))$ is an optimal function in (13), for arbitrary $C \neq 0, \sigma \neq 0$ and $\bar{x} \in \mathbb{R}^{n}$. This completes the proof of the theorem.

Remark 1. If $q=1$ and $p=s$, then assumption (11) reads as $1<p<n$ if $n>1$, and $p>1$ if $n=1$. Then, inequality (13) is the $L^{p}$ Nash's inequality

$$
\begin{aligned}
&\left(\int_{\mathbb{R}^{n}}|u(x)|^{p} \mathrm{~d} x\right)^{1+\frac{p}{n(p-1)}} \\
& \leq\left(K_{\text {opt }}\right)^{p+\frac{p^{2}}{n(p-1)}} \int_{\mathbb{R}^{n}}|\nabla u(x)|^{p} \mathrm{~d} x\left(\int_{\mathbb{R}^{n}}|u(x)| \mathrm{d} x\right)^{\frac{p^{2}}{n(p-1)}} .
\end{aligned}
$$

\section{Extremals in Gagliardo-Nirenberg/Nash's inequalities}

In this section, we find explicitly a minimizer $u_{\infty}$ of the variational problem (2) by solving the $p$-Laplacian type equation (3), which is the Euler-Lagrange equation to problem (2). Throughout the section, we assume that $n, p, q$ and $s$ satisfy

$$
\begin{array}{llclc}
1<p<n & \text { and } & 1 \leq q<s<\bar{p}:=\frac{n p}{n-p} & \text { if } & n>1, \\
p>1 & \text { and } & 1 \leq q<s<\infty & \text { if } & n=1 .
\end{array}
$$

Consider the variational problem

$$
\inf \left\{E(u):=\frac{1}{p} \int_{\mathbb{R}^{n}}|\nabla u|^{p} \mathrm{~d} x+\frac{1}{q} \int_{\mathbb{R}^{n}}|u|^{q} \mathrm{~d} x: u \in D^{p, q}\left(\mathbb{R}^{n}\right),\|u\|_{L^{s}\left(\mathbb{R}^{n}\right)}=1\right\} .
$$

Under assumption (23), one shows that (24) has a minimizer $u_{\infty}$ which can be chosen non-negative, radially-symmetric, nonincreasing and tends to 0 as $|x|$ tends to $\infty$ (see [8]). Therefore, $u_{\infty}$ can be written as,

$$
u_{\infty}(x)=v(r), \quad r:=|x|
$$


where $v:[0, \infty) \rightarrow[0, \infty)$ is a nonincreasing function which satisfies $\lim _{r \rightarrow \infty} v(r)=$ 0 . Furthermore, $u_{\infty}$ is the unique solution [13] of the Euler-Lagrange equation (3) associated to problem (24). Up to replacing $u_{\infty}$ by the rescaled function

$$
\bar{u}_{\infty}(x)=\lambda^{\frac{1}{s-q}} u_{\infty}\left(\lambda^{\frac{q-p}{p(s-q)}} x\right),
$$

we can assume, without loss of generality, that $\lambda=1$, i.e., $u_{\infty}$ uniquely solves the $p$-Laplacian type equation

$$
-\operatorname{div}\left(|\nabla u|^{p-2} \nabla u\right)+u^{q-1}=u^{s-1} .
$$

Combining (25) and (27), we obtain by a direct computation that $v$ is the solution of the ODE

$$
(p-1)\left|v^{\prime}(r)\right|^{p-2} v^{\prime \prime}(r)+\frac{n-1}{r}\left|v^{\prime}(r)\right|^{p-2} v^{\prime}(r)-v(r)^{q-1}+v(r)^{s-1}=0 .
$$

We infer from (28) that $\lim _{r \rightarrow \infty} v^{\prime}(r)$ exists because $v^{\prime \prime}(r) \geq 0$ for $r$ large enough (and hence $v^{\prime}(r)$ is nondecreasing for such $r$ ), since $v^{\prime}(r) \leq 0, \lim _{r \rightarrow \infty} v(r)=0$ and $q<s$. Moreover $\lim _{r \rightarrow \infty} v^{\prime}(r)=0$ because

$$
n \omega_{n} \int_{0}^{\infty} r^{n-1}\left|v^{\prime}(r)\right|^{p} \mathrm{~d} r=\left\|\nabla u_{\infty}\right\|_{p}^{p} \leq p E\left(u_{\infty}\right)<\infty,
$$

otherwise $\left\|\nabla u_{\infty}\right\|_{p}^{p}=\infty$ as we could find some $\epsilon>0$ such that $\left|v^{\prime}(r)\right|>\epsilon$ for all $r>n$; here $\omega_{n}$ denotes the Lebesgue measure of the unit ball in $\mathbb{R}^{n}$, and we assume that $v$ is positive everywhere. If $v$ has a compact support, then $v(r)=0=v^{\prime}(r)$ outside its support, and $\lim _{r \rightarrow \infty} v^{\prime}(r)=0$ is trivial.

Now, let $p^{*}:=p /(p-1)$, and consider the change of function

$$
H(v(r))=\frac{r^{p^{*}}}{p^{*}}
$$

for some $C^{2}$-function $H$ defined on $(0, v(0))$. As explained in the introduction, this change of function is suggested by the link existing between certain GagliardoNirenberg inequalities and mass transport theory [6]. For more details on this choice, we refer to [1] where it is explained in full details when $p=2$. Note that since by definition $H=(v \circ g)^{-1}$ with $g(t)=\left(p^{*} t\right)^{1 / p^{*}}$, then $H$ is well defined provided that $v$ is invertible. But according to Serrin-Tang [13], $v$ is positive everywhere on $[0, \infty)$ if $q \geq p$, while it has a compact support $[0, R]$ if $q<p$. Therefore, if $q \geq p$, then $H$ is well defined as a function from $(0=v(\infty), v(0))$ to $(0, \infty)$, but if $q<p$, then $H$ is a function from $(0=v(R), v(0))$ to $\left(0, R^{p^{*}} / p^{*}\right)$.

Now, we state the main theorem of this paper. It establishes an ODE for the function $H$, from which the minimizer $u_{\infty}$ of problem (24) can be computed explicitly.

Theorem 3.1. Assume that $n, p, q$ and $s$ satisfy (23). Let $H$ be a $C^{2}$-function on $(0, v(0))$ satisfying $H(v(r))=\frac{r^{p^{*}}}{p^{*}}, \frac{1}{p}+\frac{1}{p^{*}}=1$, where $v$ is a non-negative, 
nonincreasing, $C^{2}$-solution of

$$
(p-1)\left|v^{\prime}(r)\right|^{p-2} v^{\prime \prime}(r)+\frac{n-1}{r}\left|v^{\prime}(r)\right|^{p-2} v^{\prime}(r)-v(r)^{q-1}+v(r)^{s-1}=0
$$

with $\lim _{r \rightarrow \infty} v(r)=0$ and $\lim _{r \rightarrow \infty} v^{\prime}(r)=0$. Then $H(t)$ satsfies the ODE

$$
\begin{aligned}
p\left|H^{\prime}(t)\right|^{p-2} H^{\prime \prime}(t) & \left(\frac{t^{q}}{q}-\frac{t^{s}}{s}\right)+\left|H^{\prime}(t)\right|^{p-2} H^{\prime}(t)\left(t^{q-1}-t^{s-1}\right) \\
& -p(n-1)\left|H^{\prime}(t)\right|^{p-2} H^{\prime \prime}(t) \int_{0}^{t} \frac{d \tau}{\left|H^{\prime}(\tau)\right|^{p-2} H^{\prime}(\tau)}=n,
\end{aligned}
$$

for all $t \in(0, v(0))$. Therefore, if $G$ is a $C^{2}$-function on $(0, v(0))$ such that

$$
G^{\prime}(t)=\frac{\left|H^{\prime}(t)\right|^{p-2} H^{\prime}(t)}{p-1}
$$

then $G^{\prime}$ solves the first order nonlinear $O D E$

$$
p\left(\frac{t^{q}}{q}-\frac{t^{s}}{s}\right) G^{\prime \prime}(t)+(p-1)\left(t^{q-1}-t^{s-1}\right) G^{\prime}(t)-\frac{p(n-1)}{p-1} G^{\prime \prime}(t) \int_{0}^{t} \frac{d \tau}{G^{\prime}(\tau)}=n .
$$

Proof. Differentiating $H(v(r))=\frac{r^{p^{*}}}{p^{*}}$ with respect to $r$, we have that $H^{\prime}(v(r))=$ $\frac{r^{p^{*}-1}}{v^{\prime}(r)}$, that is,

$$
r=\left[H^{\prime}(v(r)) v^{\prime}(r)\right]^{\frac{1}{p^{*}-1}}=\left[H^{\prime}(v(r)) v^{\prime}(r)\right]^{p-1} .
$$

Then $H^{\prime}(t) \leq 0$ for all $t \in(0, v(0))$ because $v$ is nonincreasing on $(0, \infty)$. Differentiating again (35) with respect to $r$, we have that

$$
H^{\prime}(v)^{p-2} H^{\prime \prime}(v)\left(v^{\prime}\right)^{p}+H^{\prime}(v)^{p-1}\left(v^{\prime}\right)^{p-2} v^{\prime \prime}=\frac{1}{p-1} .
$$

We use that $v^{\prime}(r) \leq 0$ and $H^{\prime}(v) \leq 0$ to rewrite (36) as

$$
\left|H^{\prime}(v)\right|^{p-2} H^{\prime \prime}(v)\left|v^{\prime}\right|^{p}+\left|H^{\prime}(v)\right|^{p-2} H^{\prime}(v)\left|v^{\prime}\right|^{p-2} v^{\prime \prime}=\frac{1}{p-1} .
$$

To prove (31), we will express $\left|v^{\prime}\right|^{p-2} v^{\prime \prime}$ and $\left|v^{\prime}\right|^{p}$ in terms of $v$ and $H(v)$ in (37). Indeed, from (30), we have that

$$
\left|v^{\prime}\right|^{p-2} v^{\prime \prime}=\frac{-1}{p-1}\left(\frac{n-1}{r}\left|v^{\prime}\right|^{p-2} v^{\prime}-v^{q-1}+v^{s-1}\right),
$$

and from (35), we read that

$$
\frac{\left|v^{\prime}\right|^{p-2} v^{\prime}}{r}=\frac{1}{\left|H^{\prime}(v)\right|^{p-2} H^{\prime}(v)} .
$$

Combining (38) and (39), we obtain that

$$
\left|v^{\prime}\right|^{p-2} v^{\prime \prime}=\frac{-1}{p-1}\left(\frac{n-1}{\left|H^{\prime}(v)\right|^{p-2} H^{\prime}(v)}-v^{q-1}+v^{s-1}\right) .
$$


Next, we multiply (40) by $v^{\prime}$ and integrate the resulting equality over $(r, \infty)$. Noticing that $\frac{d}{d r}\left(\frac{\left|v^{\prime}(r)\right|^{p}}{p}\right)=\left|v^{\prime}\right|^{p-2} v^{\prime} v^{\prime \prime}, \lim _{r \rightarrow \infty} v(r)=0$ and $\lim _{r \rightarrow \infty} v^{\prime}(r)=0$, we have that

i.e.

$$
\left|v^{\prime}\right|^{p}=\frac{p}{p-1}\left(\frac{v^{q}}{q}-\frac{v^{s}}{s}+(n-1) \int_{r}^{\infty} \frac{v^{\prime}(r)}{\left|H^{\prime}(v(r))\right|^{p-2} H^{\prime}(v(r))} \mathrm{d} r\right),
$$

$$
\left|v^{\prime}\right|^{p}=\frac{p}{p-1}\left(\frac{v^{q}}{q}-\frac{v^{s}}{s}-(n-1) \int_{0}^{v(r)} \frac{\mathrm{d} \tau}{\left|H^{\prime}(\tau)\right|^{p-2} H^{\prime}(\tau)}\right),
$$

where we use the substitution $\tau=v(r)$ in the integral term. Inserting (40) and (41) into (37), we obtain

$$
\begin{aligned}
p\left|H^{\prime}(v)\right|^{p-2} H^{\prime \prime}(v) & \left(\frac{v^{q}}{q}-\frac{v^{s}}{s}\right)+\left|H^{\prime}(v)\right|^{p-2} H^{\prime}(v)\left(v^{q-1}-v^{s-1}\right) \\
& \quad-p(n-1)\left|H^{\prime}(v)\right|^{p-2} H^{\prime \prime}(v) \int_{0}^{v(r)} \frac{\mathrm{d} \tau}{\left|H^{\prime}(\tau)\right|^{p-2} H^{\prime}(\tau)}=n,
\end{aligned}
$$

that is (31). Finally, setting $G^{\prime}(t)=\frac{\left|H^{\prime}(t)\right|^{p-2} H^{\prime}(t)}{p-1}$, we have that $G^{\prime \prime}(t)=$ $\left|H^{\prime}(t)\right|^{p-2} H^{\prime \prime}(t)$, and then (31) reads as (34). This completes the proof of the theorem.

Remark 2. Using that $H(v(r))=\frac{r^{p^{*}}}{p^{*}}$ and $H^{\prime}(v(r))=\frac{r^{p^{*}-1}}{v^{\prime}(r)}$, we have:

(i) if $q \geq p$ in which case $H:(0=v(\infty), v(0)) \rightarrow(0, \infty)$, then $\lim _{t \rightarrow 0^{+}} H(t)=\infty$ and $\lim _{t \rightarrow 0^{+}} H^{\prime}(t)=-\infty$, because $v(\infty)=v^{\prime}(\infty)=0$.

(ii) if $q<p$ in which case $H:(0=v(R), v(0)) \rightarrow\left(0, R^{p^{*}} / p^{*}\right)$, then $\lim _{t \rightarrow 0^{+}} H(t)=$ $R^{p^{*}} / p^{*} \in(0, \infty)$ and $\lim _{t \rightarrow 0^{+}} H^{\prime}(t)=-\infty$, because $v(R)=v^{\prime}(R)=0$.

When the space dimension is $n=1$, then ODE (34) reduces to the linear first order ODE in $G^{\prime}(t)$,

$$
p\left(\frac{t^{q}}{q}-\frac{t^{s}}{s}\right) G^{\prime \prime}(t)+(p-1)\left(t^{q-1}-t^{s-1}\right) G^{\prime}(t)=1,
$$

which can be solved explicitly. Therefore, we obtain explicitly the minimizer $u_{\infty}$ to the variational problem (24) via (25), (26), (29), (33) and (42). And Theorem 2.1 gives explicitly the best constant $K_{o p t}$ and optimal functions $u_{\sigma, \bar{x}}(x)=C u_{\infty}(\sigma(x-$ $\bar{x})$ ) of all the Gagliardo-Nirenberg/Nash's inequalities when $n=1$. The results are summarized in the next corollary.

Corollary 3.2. If $n=1, p>1$ and $1 \leq q<s<\infty$, then

$$
G^{\prime}(t)=\frac{1}{p\left|\frac{t^{q}}{q}-\frac{t^{s}}{s}\right|^{\frac{1}{p^{*}}}} \int \frac{d t}{\operatorname{sign}\left(\frac{t^{q}}{q}-\frac{t^{s}}{s}\right)\left|\frac{t^{q}}{q}-\frac{t^{s}}{s}\right|^{\frac{1}{p}}}
$$

is a solution of ODE (34). Therefore, the minimizer $u_{\infty}$ to problem (24) can be computed explicitly via (25), (26), (29) and (33), and Theorem 2.1 gives the best constant $K_{\text {opt }}$ and optimal functions $u_{\sigma, \bar{x}}(x)=C u_{\infty}(\sigma(x-\bar{x}))$ of all the 
Gagliardo-Nirenberg/Nash's inequalities (13) when $n=1, p>1$ and $1 \leq q<s<$ $\infty$.

Proof. If $n=1$, then (34) becomes the first order linear ODE in $G^{\prime}(t)$,

$$
G^{\prime \prime}(t)+\frac{(p-1)\left(t^{q-1}-t^{s-1}\right)}{p\left(\frac{t^{q}}{q}-\frac{t^{s}}{s}\right)} G^{\prime}(t)=\frac{1}{p\left(\frac{t^{q}}{q}-\frac{t^{s}}{s}\right)},
$$

whose solution is

$$
G^{\prime}(t)=e^{-\int A(t) \mathrm{d} t} \int\left[B(t) e^{\int A(t) \mathrm{d} t}\right] \mathrm{d} t
$$

where

$$
A(t)=\frac{(p-1)\left(t^{q-1}-t^{s-1}\right)}{p\left(\frac{t^{q}}{q}-\frac{t^{s}}{s}\right)} \quad \text { and } \quad B(t)=\frac{1}{p\left(\frac{t^{q}}{q}-\frac{t^{s}}{s}\right)} .
$$

It is easy to check that

$$
\int A(t) \mathrm{d} t=\ln \left(\left|\frac{t^{q}}{q}-\frac{t^{s}}{s}\right|^{1 / p^{*}}\right)
$$

and

$$
B(t) e^{\int A(t) \mathrm{d} t}=\frac{\left|\frac{t^{q}}{q}-\frac{t^{s}}{s}\right|^{1 / p^{*}}}{p\left(\frac{t^{q}}{q}-\frac{t^{s}}{s}\right)}=\frac{1}{p \operatorname{sign}\left(\frac{t^{q}}{q}-\frac{t^{s}}{s}\right)\left|\frac{t^{q}}{q}-\frac{t^{s}}{s}\right|^{1 / p}} .
$$

Inserting (46) and (47) into (45) we conclude (43).

For applications of Corollary 3.2, we refer to [1] where the minimizer $u_{\infty}$ is evaluated explicitly in some specific examples when $p=2$.

Notice that if $q=1$ and $p=s$, our analysis allows to obtain the best constant and optimal functions of all the $L^{p}$ Nash's inequalities (22), at least on the real line, $n=1$. For illustration, we consider the $L^{2}$ Nash's inequality, i.e., $q=1$ and $p=s=2$, though in this case, the result could be obtained in a simpler way.

Corollary 3.3. If $n=1, q=1$ and $p=s=2$, then

$$
H(t)=2\left(\arcsin \sqrt{\frac{2-t}{2}}\right)^{2} \chi_{[t \leq 2]}(t)-2\left(\operatorname{arcsinh} \sqrt{\frac{t-2}{2}}\right)^{2} \chi_{[t>2]}(t)
$$

is a solution of ODE (31). Then the sharp Nash's inequality

$$
\left(\int_{\mathbb{R}}|u(x)|^{2} d x\right)^{3} \leq\left(K_{\text {opt }}\right)^{6} \int_{\mathbb{R}}|\nabla u(x)|^{2} d x\left(\int_{\mathbb{R}}|u(x)| d x\right)^{4}
$$

holds, with the best constant $K_{\text {opt }}$ computable by Theorem 2.1, and the optimal functions given by $u_{\sigma, \bar{x}}(x)=C u_{\infty}(\sigma(x-\bar{x}))$ where

$$
u_{\infty}(x)=(1+\cos |x|) \chi_{[|x| \leq \pi]}(x),
$$

and $C \neq 0, \sigma \neq 0$ and $\bar{x} \in \mathbb{R}$ are arbitrary. 
Proof. If $q=1$ and $p=s=2$, then (43) reads as

$$
H^{\prime}(t)=G^{\prime}(t)=\frac{1}{\sqrt{\left|2 t-t^{2}\right|}} \int \frac{\mathrm{d} t}{\operatorname{sign}\left(2 t-t^{2}\right) \sqrt{\left|2 t-t^{2}\right|}} .
$$

We integrate (51) to have that

$$
H(t)=\frac{1}{2}\left(\int \frac{\mathrm{d} t}{\sqrt{2 t-t^{2}}}\right)^{2} \chi_{[t \leq 2]}(t)-\frac{1}{2}\left(\int \frac{\mathrm{d} t}{\sqrt{t^{2}-2 t}}\right)^{2} \chi_{[t>2]}(t)+\gamma,
$$

i.e.,

$$
H(t)=2\left(\arcsin \sqrt{\frac{2-t}{2}}\right)^{2} \chi_{[t \leq 2]}(t)-2\left(\operatorname{arcsinh} \sqrt{\frac{t-2}{2}}\right)^{2} \chi_{[t>2]}(t)+\gamma,
$$

for some constant $\gamma$. Now, we solve for $v(r)$ in the equation $H(v(r))=\frac{r^{2}}{2}$. We argue that if the set $[v(r)>2]$ were nonempty, then on this set, we would have that

$$
H(v(r))=-2\left(\operatorname{arcsinh} \sqrt{\frac{v(r)-2}{2}}\right)^{2}+\gamma=\frac{r^{2}}{2},
$$

that is,

$$
v(r)=2\left[1+\sinh ^{2}\left(\frac{1}{2} \sqrt{2 \gamma-r^{2}}\right)\right]=2 \cosh ^{2}\left(\frac{1}{2} \sqrt{2 \gamma-r^{2}}\right) .
$$

Inserting (53) into ODE (30), we have that $\gamma=0$, from which we deduce a contradiction since $\sqrt{2 \gamma-r^{2}}$ would not make sense. Therefore $[v(r)>2]$ is empty, and then $[v(r) \leq 2]=\mathbb{R}$. Hence, $H(v(r))=\frac{r^{2}}{2}$ reads as

$$
H(v(r))=2\left(\arcsin \sqrt{\frac{2-v(r)}{2}}\right)^{2}+\gamma=\frac{r^{2}}{2},
$$

i.e.

$$
\arcsin \sqrt{\frac{2-v(r)}{2}}=\frac{1}{2} \sqrt{r^{2}-2 \gamma} \leq \frac{\pi}{2},
$$

where the last inequality follows from the definition of the function arcsin. Then,

$$
\begin{aligned}
v(r) & =2\left[1-\sin ^{2}\left(\frac{1}{2} \sqrt{r^{2}-2 \gamma}\right)\right] \chi_{\left[r^{2} \leq \pi^{2}+2 \gamma\right]}(r) \\
& =\left[1+\cos \left(\sqrt{r^{2}-2 \gamma}\right)\right] \chi_{\left[r^{2} \leq \pi^{2}+2 \gamma\right]}(r) .
\end{aligned}
$$

We insert (54) into (30) to have that $\gamma=0$. Therefore, (52) proves (48), and (54) gives that

$$
u_{\infty}(x)=v(|x|)=(1+\cos |x|) \chi_{[|x| \leq \pi]}(x) .
$$

Finally, we use Theorem 2.1 to deduce that the sharp Nash's inequality (49) holds with the best constant $K_{\text {opt }}$ given by (14)-(15), and the optimal functions are $u_{\sigma, \bar{x}}(x)=C u_{\infty}(\sigma(x-\bar{x}))$ where $u_{\infty}$ is defined by $(55)$. 
When $n \geq 2$, we have not been able to explicitly solve ODE (34) in all generalities due to the nonlinear term $\frac{p(n-1)}{p-1} G^{\prime \prime}(t) \int_{0}^{t} \frac{\mathrm{d} \tau}{G^{\prime}(\tau)}$. But if we furthermore assume that $G^{\prime \prime}(t) \int_{0}^{t} \frac{\mathrm{d} \tau}{G^{\prime}(\tau)}$ is a constant, then (34) reduces to a linear ODE that can be solved explicitly. As a consequence, we obtain the sharp constants and optimal functions of the Gagliardo-Nirenberg inequalities (13) when $q=p^{*}(s-1)$ or $q=1+\frac{s}{p^{*}}$. These results recover previous results obtained by Del-Pino and Dolbeault in [8].

Corollary 3.4. Under the assumptions of Theorem 3.1, assume furthermore that

$$
G^{\prime \prime}(t) \int_{0}^{t} \frac{d \tau}{G^{\prime}(\tau)}=k
$$

where $k$ is a constant. Then $G$ solves $O D E$ (34) if and only if $q=1+\frac{s}{p^{*}}$ or $q=p^{*}(s-1)$. Therefore,

(i) If $q=1+\frac{s}{p^{*}}$, then

$$
H(t)=\frac{p}{s-p}\left(\frac{n p-s(n-p)}{s-p}\right)^{1 / p-1} t^{\frac{p-s}{p}}-\gamma
$$

is a solution of ODE (31), for some constant $\gamma$. Then the sharp GagliardoNirenberg inequality

$$
\|u\|_{s} \leq K_{\text {opt }}\|\nabla u\|_{p}^{\theta}\|u\|_{1+\frac{s}{p^{*}}}^{1-\theta} \quad \forall u \in D^{p, q}\left(\mathbb{R}^{n}\right)
$$

holds with $\theta=\frac{n p(s-q)}{s[n p-q(n-p)]}$, the best constant $K_{\text {opt }}$ computable by Theorem 2.1, and the optimal functions given by $u_{\sigma, \bar{x}}(x)=C u(\sigma(x-\bar{x}))$ where

$$
u(x)=\frac{1}{\left(1+|x|^{p^{*}}\right)^{\frac{p}{s-p}}},
$$

and $C \neq 0, \sigma \neq 0$ and $\bar{x} \in \mathbb{R}^{n}$ are arbitrary.

(ii) If $q=p^{*}(s-1)$, then

$$
H(t)=-\frac{p-1}{p-s}\left(\frac{(n-1)(p-s)+s(p-1)}{p-s}\right)^{1 / p-1} t^{\frac{p-s}{p-1}}+\gamma
$$

is a solution of ODE (31), for some constant $\gamma$. Then the sharp GagliardoNirenberg inequality

$$
\|u\|_{s} \leq K_{\text {opt }}\|\nabla u\|_{p}^{\theta}\|u\|_{p^{*}(s-1)}^{1-\theta} \quad \forall u \in D^{p, q}\left(\mathbb{R}^{n}\right)
$$

holds with $\theta=\frac{n p(s-q)}{s[n p-q(n-p)]}$, the best constant $K_{\text {opt }}$ computable by Theorem 2.1, and the optimal functions given by $u_{\sigma, \bar{x}}(x)=C u(\sigma(x-\bar{x}))$ where

$$
u(x)=\left(1-|x|^{p^{*}}\right)_{+}^{\frac{p-1}{p-s}},
$$

and $C \neq 0, \sigma \neq 0$ and $\bar{x} \in \mathbb{R}^{n}$ are arbitrary. 
Proof. With $G^{\prime \prime}(t) \int_{0}^{t} \frac{\mathrm{d} \tau}{G^{\prime}(\tau)}=k$, ODE (34) reads as

$$
p\left(\frac{t^{q}}{q}-\frac{t^{s}}{s}\right) G^{\prime \prime}(t)+(p-1)\left(t^{q-1}-t^{s-1}\right) G^{\prime}(t)=n+\frac{p(n-1)}{p-1} k .
$$

Set $\phi(t)=\int_{o}^{t} \frac{\mathrm{d} \tau}{G^{\prime}(\tau)}$. Then $G^{\prime \prime}(t) \int_{0}^{t} \frac{\mathrm{d} \tau}{G^{\prime}(\tau)}=k$ gives that

$$
\frac{\phi^{\prime \prime}(t)}{\phi^{\prime}(t)}=-k \frac{\phi^{\prime}(t)}{\phi(t)}
$$

that is,

$$
\phi^{\prime}(t) \phi(t)^{k}=A,
$$

for some constant $A$. Note that $k \neq-1$, if not, $\phi(t)=\phi(0) e^{A t}=0$ which cannot hold because $G^{\prime}$ (and therefore $H^{\prime}$ ) is not constant. So, integrating (64), we have that $\phi(t)=(A(k+1) t)^{1 / k+1}$, that is,

$$
G^{\prime}(t)=B t^{\frac{k}{k+1}},
$$

where $B=\frac{(A(k+1))^{k / k+1}}{A}$. We insert (65) into (63) to have that

$$
\left(\frac{p k}{q(k+1)}+p-1\right) t^{q-\frac{1}{k+1}}-\left(p-1+\frac{p k}{s(k+1)}\right) t^{s-\frac{1}{k+1}}=\frac{n+\frac{p(n-1)}{p-1} k}{B},
$$

for all $t \in(0, v(0))$. Since $q \neq s$, then (66) holds for all $t$ if and only if

$$
q-\frac{1}{k+1}=0 \quad \text { and } \quad p-1+\frac{p k}{s(k+1)}=0,
$$

or

$$
p-1+\frac{p k}{q(k+1)}=0 \quad \text { and } \quad s-\frac{1}{k+1}=0 .
$$

It is easy to see that (67) gives $q=1+\frac{s}{p^{*}}$, while (68) gives that $q=p^{*}(s-1)$. Now, assume that $q=1+\frac{s}{p^{*}}$. By direct computations, (66) and (67) give that

$$
B=\frac{n+\frac{p(n-1)}{p-1} k}{\frac{p k}{q(k+1)}+p-1}=-\frac{n p-s(n-p)}{(p-1)(s-p)} .
$$

Observe that $B<0$ because $p<s<\frac{n p}{n-p}$ due to assumption (23) and the fact that $q=1+\frac{s}{p^{*}}$. Then using (65), (33) and the fact that $H^{\prime}(t) \leq 0$ and $p>1$, we have that

$$
H^{\prime}(t)=-((1-p) B)^{\frac{1}{p-1}} t^{-\frac{s}{p}} .
$$

We integrate (70), and we use (69) to have that

$$
H(t)=\frac{p((1-p) B)^{\frac{1}{p-1}}}{s-p} t^{\frac{p-s}{p}}-\gamma=\frac{p}{s-p}\left(\frac{n p-s(n-p)}{s-p}\right)^{\frac{1}{p-1}} t^{\frac{p-s}{p}}-\gamma
$$


where $\gamma$ is a constant. This proves (57). Next, we solve for $v(r)$ in the equation $H(v(r))=\frac{r^{p^{*}}}{p^{*}}$. Using $(71)$, we have that

$$
v(r)=\frac{\left(\frac{1}{p^{*} \delta}\right)^{\frac{p}{p-s}}}{\left(\gamma p^{*}+r^{p^{*}}\right)^{\frac{p}{s-p}}}, \quad \delta:=\frac{p}{s-p}\left(\frac{n p-s(n-p)}{s-p}\right)^{\frac{1}{p-1}} .
$$

Then

$$
u_{\infty}(x)=v(|x|)=\frac{\left(\frac{1}{p^{*} \delta}\right)^{\frac{p}{p-s}}}{\left(\gamma p^{*}+|x|^{p^{*}}\right)^{\frac{p}{s-p}}}
$$

solves PDE (27), where $\gamma$ is uniquely determined by the normalization constraint $\|u\|_{s}=1$. Using Theorem 2.1, we deduce that the sharp Gagliardo-Nirenberg inequality (58) holds with the best constant $K_{\text {opt }}$ given by (14)-(15), and the optimal functions are $u_{\sigma, \bar{x}}(x)=C u_{\infty}(\sigma(x-\bar{x}))$. Now we rescale $u_{\infty}(x)$ defined by (73), to conclude that $u_{\sigma, \bar{x}}(x)=C u(\sigma(x-\bar{x}))$ are optimal functions of the Gagliardo-Nirenberg inequality (58), where

$$
u(x)=\frac{1}{\left(1+|x|^{p^{*}}\right)^{\frac{p}{s-p}}} .
$$

if $q=p^{*}(s-1)$, the proof is similar. Indeed from (66) and (68), we have that

$$
B=-\frac{n+\frac{p(n-1)}{p-1} k}{\frac{p k}{s(k+1)}+p-1}=-\frac{(n-1)(p-s)+s(p-1)}{(p-1)(p-s)},
$$

which is negative since $n>1$ and $p>s$, because $q=p^{*}(s-1)<s$ from (23). (65) together with (33) give that

$$
H^{\prime}(t)=-((1-p) B)^{\frac{1}{p-1}} t^{\frac{1-s}{p-1}} .
$$

Integrating (75), we obtain after using (74),

$$
H(t)=-\frac{p-1}{p-s}\left(\frac{(n-1)(p-s)+s(p-1)}{p-s}\right)^{\frac{1}{p-1}} t^{\frac{p-s}{p-1}}+\gamma
$$

where $\gamma$ is a constant. This proves (60). As before, we solve for $v(r)$ in $H(v(r))=$ $\frac{r^{p^{*}}}{p^{*}}$. We have that

$v(r)=\left(\frac{1}{p^{*} \sigma}\right)^{\frac{p-1}{p-s}}\left(p^{*} \gamma-r^{p^{*}}\right)^{\frac{p-1}{p-s}}, \quad \sigma:=\frac{p-1}{p-s}\left(\frac{(n-1)(p-s)+s(p-1)}{p-s}\right)^{\frac{1}{p-1}}$

on its - compact - support, that is,

$$
v(r)=\left(\frac{1}{p^{*} \sigma}\right)^{\frac{p-1}{p-s}}\left(p^{*} \gamma-r^{p^{*}}\right)_{+}^{\frac{p-1}{p-s}}, \quad \forall r \geq 0 .
$$

Then

$$
u_{\infty}(x)=v(|x|)=\left(\frac{1}{p^{*} \sigma}\right)^{\frac{p-1}{p-s}}\left(p^{*} \gamma-|x|^{p^{*}}\right)_{+}^{\frac{p-1}{p-s}}
$$


solves PDE (27), where $\gamma$ is uniquely determined by the normalization constraint $\|u\|_{s}=1$. Finally using Theorem 2.1, we deduce the sharp Gagliardo-Nirenberg inequality (61), with the best constant $K_{\text {opt }}$ given by (14)-(15), and the optimal functions determined by $u_{\sigma, \bar{x}}(x)=C u(\sigma(x-\bar{x}))$, where

$$
u(x)=\left(1-|x|^{p^{*}}\right)_{+}^{\frac{p-1}{p-s}}
$$

is obtained by rescaling (78). This completes the proof of Corollary 3.4.

\section{Acknowledgements}

The author would like to thank an anonymous referee for his suggestion which improved the presentation of this work.

\section{References}

[1] M. Agueh, Sharp Gagliardo-Nirenberg inequalities and Mass transport theory, Journal of Dynamics and Differential Equations 18 (4) (2006), 1069-1093.

[2] M. Agueh, Gagliardo-Nirenberg inequalities involving the gradient $L^{2}$-norm, C. R. Math. Acad. Sci. Paris, Ser. I 346 (2008), 757-762.

[3] M. Agueh, N. Ghoussoub, and X. Kang, Geometric inequalities via a general comparison principle for interacting gases, Geom. Funct. Anal. 14 (2004), 215-244.

[4] T. Aubin. Problème isopérimétrique et espaces de Sobolev, J. Diff. Geom. 11 (1976), 573-598.

[5] E. Carlen, and M. Loss, Sharp constant in Nash's inequality, International Mathematics Research Notices. 7 (1993), 213-215.

[6] D. Cordero-Erausquin, B. Nazaret, and C. Villani. A mass transportation approach to sharp Sobolev and Gagliardo-Nirenberg inequalities, Adv. Math. 182 (2004), 307332.

[7] M. Del-Pino, and J. Dolbeault, Best constants for Gagliardo-Nirenberg inequalities and applications to nonlinear diffusions, J. Math. Pures Appl. 90, 81 (2002), 847-875.

[8] M. Del-Pino, and J. Dolbeault, The optimal Euclidean $L^{p}$-Sobolev logarithmic inequaity, J. Funct. Anal. 197 (1) (2003), 151-161.

[9] E. Gagliardo, Proprietà di alcune classi di funzioni più variabili, Ric. Mat. 7 (1958), $102-137$.

[10] E. H. Lieb, and M. Loss, Analysis, Graduate Studies in Mathematics, 14, AMS, 1997.

[11] R. J. McCann, A convexity principle for interacting gases, Adv. Math. 128 (1) (1997), $153-179$.

[12] L. Nirenberg, On elliptic partial differential equations, Ann. Sc. Norm. Pisa 13 (1959), $116-162$.

[13] J. Serrin, and M. Tang, Uniqueness of ground states for quasilinear elliptic equations, Indiana Univ. Math. J. 49 (3) (2000), 897-923.

[14] G. Talenti. Best constants in Sobolev inequality, Ann. Mat. Pura Appl. 110 (1976), $353-372$. 
Martial Agueh

Department of Mathematics and Statistics

University of Victoria

PO. Box. 3045 STN CSC

Victoria B.C., V8W 3P4

Canada

e-mail: agueh@math.uvic.ca

Received: 16 May 2007.

Revised: 19 December 2007.

Accepted: 7 January 2008. 